\title{
Ciclo de vida de duas espécies de Goeldichironomus (Diptera, Chironomidae)
}

\author{
Juliano José Corbi ${ }^{1} \&$ Susana Trivinho-Strixino ${ }^{1}$
}

\author{
${ }^{1}$ Departamento de Hidrobiologia, Laboratório de Entomologia Aquática, Universidade Federal de São Carlos, C.P. 676, 13560-970 \\ São Carlos-SP, Brasil.
}

\begin{abstract}
Life cycle of two Goeldichironomus species (Diptera, Chironomidae). Two species of Goeldichironomus were reared under laboratory conditions $\left(21^{\circ} \mathrm{C}-26^{\circ} \mathrm{C}\right)$ with food supply of TetraMin $\AA$ and Avemicina Purina $\AA$, fish food and bird food, to obtain bionomic information and to analyze culture rearing viability. The egg masses of the two species were maintained in petri dishes. The average development duration from egg to adult emergence was 28 (23-34) days for Goeldichironomus maculatus Strixino \& Strixino, 1991 and 20 (18-22) days for Goeldichironomus luridus TrivinhoStrixino \& Strixino, 2005. The larval instars of both species were determinated by the relationship between head capsule and body length sizes. These measures were used for the estimation of the growth curves and daily development rates of the species. The rearing has occurred satisfactorily, with fast growth and low mortality, but did not have copulation in laboratory conditions.
\end{abstract}

KEYWORDS. Chironomidae; egg masses; Goeldichironomus.

RESUMO. Ciclo de vida de duas espécies de Goeldichironomus (Diptera, Chironomidae). Duas espécies de Goeldichironomus foram criadas em condições de laboratório $\left(21^{\circ} \mathrm{C}-26^{\circ} \mathrm{C}\right)$, com alimento para peixes do tipo TetraMin ${ }^{\circledR}$ e alimento para aves Avemicina Purina ${ }^{\circledR}$, para se obter informações bionômicas e analisar a viabilidade de cultivo para fins de bioensaios. As massas ovígeras das duas espécies, foram mantidas em placas de Petri até a eclosão das lárvulas. A duração média do desenvolvimento larval até a emergência dos adultos foi de 28 (23-34) dias para Goeldichironomus maculatus Strixino \& Strixino, 1991 e 20 (18-22) dias para Goeldichironomus luridus Trivinho-Strixino \& Strixino, 2005. Os estádios larvais para as duas espécies foram determinados através da relação entre o tamanho da cabeça e o tamanho do corpo. Essas medidas foram usadas para determinar as curvas de crescimento e estimar a taxa de crescimento diário das duas espécies. As criações ocorreram de forma satisfatória, com rápido crescimento larval e baixa taxa de mortalidade, mas, não houve acasalamento em condições de laboratório.

PALAVRAS-CHAVE. Chironomidae; desovas; Goeldichironomus.

As larvas de Chironomidae representam um dos principais componentes da macrofauna bentônica presente nos sedimentos e na vegetação dos sistemas aquáticos continentais (Trivinho-Strixino \& Strixino 1998). Informações bionômicas, como duração do desenvolvimento larval, taxas de eclosão e de emergência são essenciais para um melhor entendimento e análise da dinâmica populacional dessas larvas. Estas informações são de difícil obtenção na natureza e, muitas vezes, prescindem de estudos autoecológicos em condições de laboratório. Tais estudos podem ainda gerar informações adicionais que podem servir de subsídios para bioensaios, como os de toxicidade utilizando essas larvas (Strixino \& Strixino 1982), uma vez que através desses estudos é possível determinar o potencial de utilização de determinadas espécies regionais em trabalhos dessa natureza.

No presente estudo são apresentadas informações a respeito do ciclo de vida de duas espécies Goeldichironomus comuns em sistemas aquáticos da região central do Estado de São Paulo, criadas em condições de laboratório a partir de massas ovígeras obtidas em campo.

Pela facilidade da criação das espécies e análise de seus povoamentos no laboratório, foi possível obter-se informações bionômicas, bem como condições de suas ocorrências.

\section{MATERIAL E MÉTODOS}

Obtenção das massas ovígeras. As massas ovígeras de Goeldichironomus luridus Trivinho-Strixino \& Strixino, 2005 foram obtidas em coletas de campo, em lagoas de estabilização situadas dentro das imediações do matadouro de Frango (Rei Frango $\left.{ }^{\circledR}\right)$, localizado no Município de São Carlos, SP, Brasil. As massas ovígeras de Goeldichironomus maculatus Strixino \& Strixino, 1991, foram coletadas na Lagoa do Boa Vista situada dentro das imediações da Fazenda Canchim (São Carlos,SP, Brasil). Os substratos contendo as desovas foram transferidos para recipientes plásticos contendo água do próprio local de coleta e, posteriormente trazidas ao laboratório.

Criação no laboratório. As desovas foram, contadas diretamente sob estereomicroscópio, desenhadas, fotografadas e colocadas em placas de Petri com água destilada até que as lárvulas tivessem eclodido e abandonado a mucilagem gelatinosa que envolve os ovos. Após esse período as larvas foram criadas em bandejas plásticas $(45 \mathrm{~cm}$ x $35 \mathrm{~cm} \mathrm{x}$ $6 \mathrm{~cm})$ contendo água (4litros) permanentemente aeradas e mantidas em temperatura ambiente $\left(21^{\circ} \mathrm{C}-26^{\circ} \mathrm{C}\right)$. As espécies foram colocadas em bandeja (um total de 20), onde foram introduzidas 100 larvas recém eclodidas, e alimentadas com 
ração de peixe do tipo TetraMin ${ }^{\circledR}$, e ração de ave do tipo Avemicina-Purina ${ }^{\circledR}$. As larvas de $G$. luridus foram retiradas diariamente de cada bandeja de criação. Em virtude do ciclo de vida ser mais longo, as larvas de G. maculatus foram retiradas dia sim e dia não das bandejas.

Determinação do ciclo de vida e dos estádios larvais. Foram medidos o comprimento do corpo e o comprimento ventral da cápsula cefálica de cada larva. Duas bandejas de criação foram cobertas por gaiolas $(40 \mathrm{~cm} \times 35 \mathrm{~cm} \times 35 \mathrm{~cm})$ que permitiram a captura dos adultos emergidos. As larvas retiradas foram observadas em estereomicroscópio, fixadas em álcool a 70\% e, posteriormente, medidas em microscópio óptico com ocular micrométrica, onde foi obtida a curva de crescimento através do tamanho de corpo em relação ao tempo (dias) e as fases larvais (estádios) determinadas relacionando-se o comprimento do corpo e o comprimento ventral da cápsula cefálica. As curvas de crescimento foram obtidas segundo modelo proposto por Krüger (1973).

Regra de Dyar. A regra de Dyar determina a "razão de crescimento" (r). Essa regra tem sido amplamente aplicada nos artrópodes (Ford 1959) e, além disso, é utilizada em diversos estudos sobre a história da vida de Chironomidae (Strixino 1973).

\section{RESULTADOS E DISCUSSÃO}

Oviposição, Massas Ovígeras e desenvolvimento embrionário: As massas ovígeras de Chironomidae são constituídas por uma matriz gelatinosa protetora dos ovos que, quando em contato com a água, incha-se, dando à desova um aspecto característico. O formato da massa gelatinosa é em geral característico para determinados grupos (Nolte 1993).
As massas ovígeras das espécies de Goeldichironomus são de formato plano, mais ou menos triangulares, contendo no seu interior fileira(s) de ovos dispostos em "loops" (Fittkau 1965; Trivinho-Strixino \& Strixino 1998). As duas espécies aqui analisadas apresentam o mesmo tipo de disposição (Fig. 1 e 2). Em G. maculatus as massas ovígeras apresentam cerca de 780 ovos com forma globular achatada na porção que adere ao substrato. As desovas de G. luridus possuem em torno de 600 ovos, com tonalidade esverdeada e aspecto tubular alongada. Após a oviposição, os ovos passam por um período de desenvolvimento comum a família Chironomidae, na qual, sofrem uma série de divisões nucleares (Sander 2000) (Fig. 2). Em ambas espécies, 48 horas após a oviposição, as lárvulas incolores do estádio I começam a eclodir dos ovos, onde permanecem por mais um período de 12 horas nesse processo. Após esse período, as larvas abandonam a massa gelatinosa que envolve os ovos e, a seguir nadam ativamente, dispersando-se.

\section{Ciclo de vida}

Goeldichironomus maculatus

A duração média do desenvolvimento de G. maculatus é de 28 dias (23 a 34). Ao eclodir das massas ovígeras as larvas medem cerca de $900 \mu \mathrm{m}$ e o seu tamanho máximo é de $9459 \mu \mathrm{m}$. O crescimento em comprimento de G. maculatus (relação comprimento total/idade das larvas) é praticamente contínuo (Fig. 3). O ciclo de vida de G. maculatus constitui-se em 4 estádios bem diferenciados: I estádio (6 dias), o II estádio (3 dias), o III (5 dias) e o IV estádio (14 dias) (Fig. 5B e Tab. IB). A aplicação da regra de Dyar evidenciou uma razão de crescimento constante para os estádios larvais com $\mathrm{r}=1,75 \mu \mathrm{m}$.
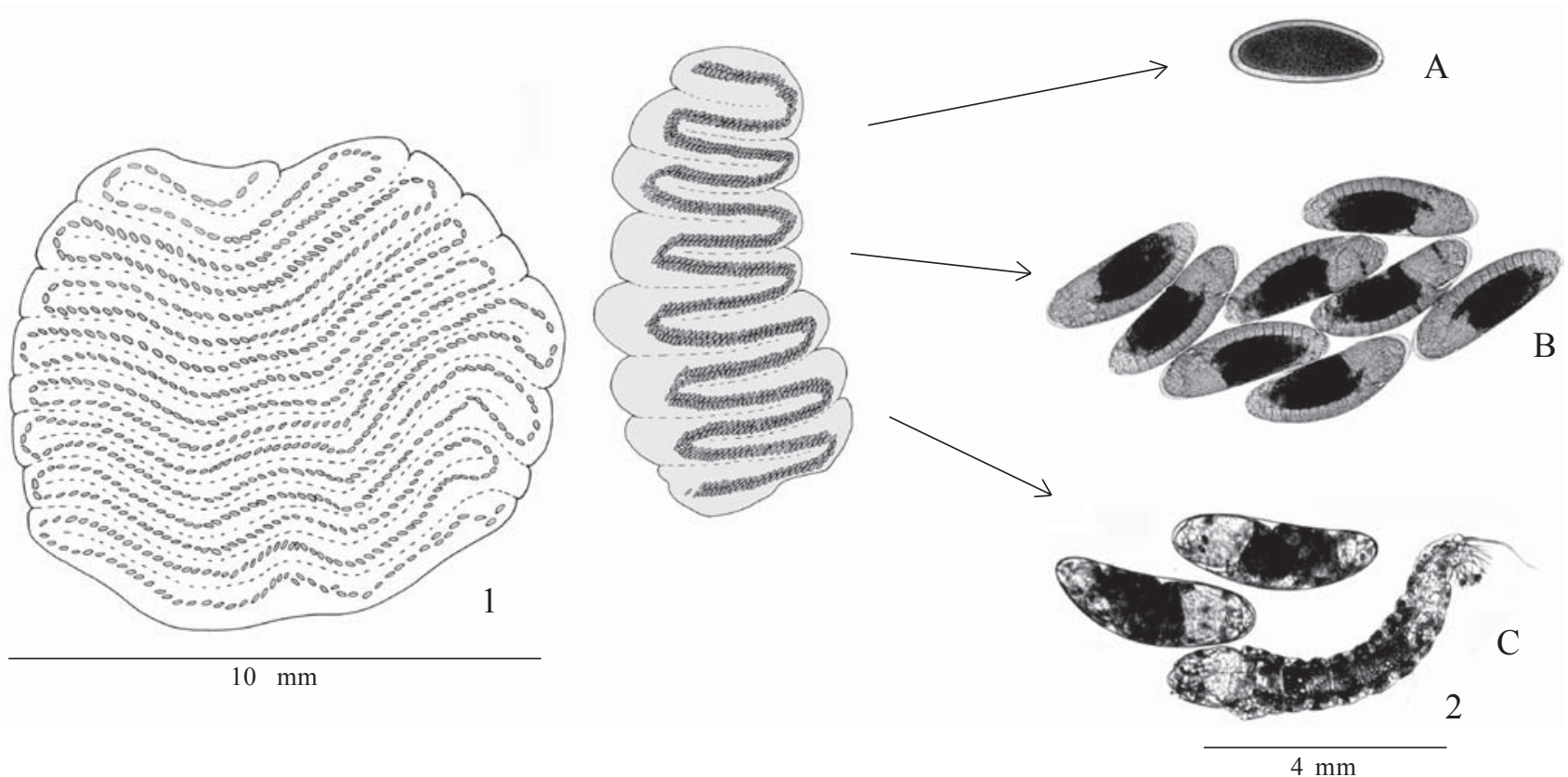

Figs. 1-2. 1, Desova de Goeldichironomus luridus Trivinho-Strixino \& Strixino; 2, Desova de Goledichironomus maculatus Strixino \& Strixino; (A) Ovo após 7 horas de desenvolvimento; (B) Ovos após 30 horas de desenvolvimento; (C) Ovos após 42 horas de desenvolvimento. 


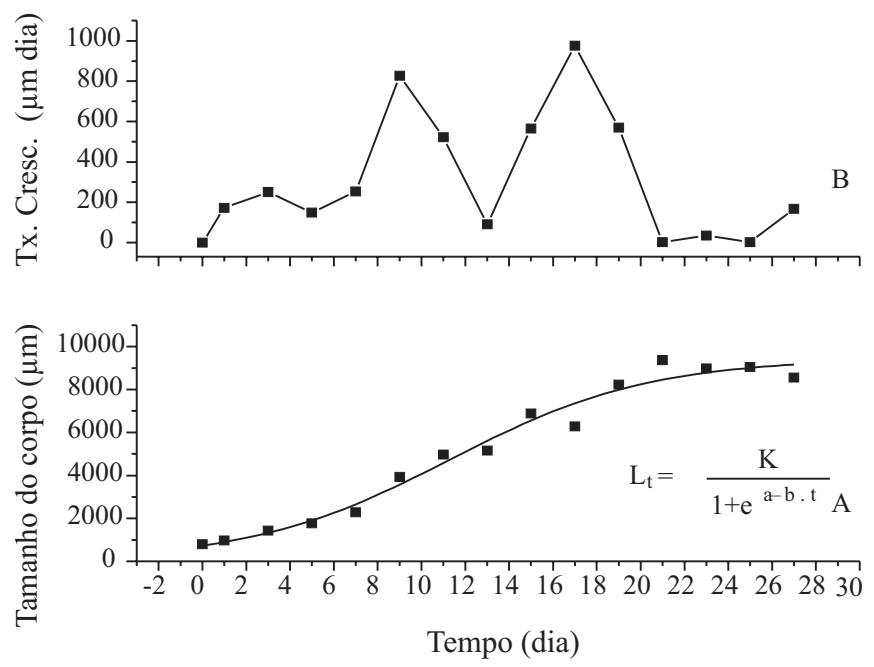

Fig. 3. Goeldichironomus maculatus Strixino \& Strixino. A. Curva de crescimento da população nas condições de laboratório $\left(21-26^{\circ} \mathrm{C}\right)$; $\mathrm{B}$. Taxa de crescimento diário das larvas $\left(\mathrm{n}_{2}-\mathrm{n}_{1} / \mathrm{t}_{2}-\mathrm{t}_{1}\right)$. Lt (comprimento médio no instante $\mathrm{t}$ ); $\mathrm{K}$ (tamanho máximo alcançado pelas larvas); $\mathrm{t}$ (tempo em dias); a e b são constantes.

\section{Goeldichironomus luridus}

A duração média do ciclo de vida de G. luridus é de 20 dias (18 a 22 dias) e as larvas eclodem dos ovos em um período máximo de dois dias. A partir desse tempo as larvas se desenvolvem nas bandejas, com um crescimento praticamente contínuo atingindo a fase adulta em um período de 18 dias. Ao eclodir, as lárvulas medem $510 \mu \mathrm{m}$ e o seu tamanho máximo é de $5080 \mu \mathrm{m}$. O crescimento em comprimento de G. luridus (relação comprimento total/idade das larvas) nas condições de laboratório é praticamente contínuo (Fig. 4). As larvas de $G$. luridus apresentam tempos diferentes de duração para os
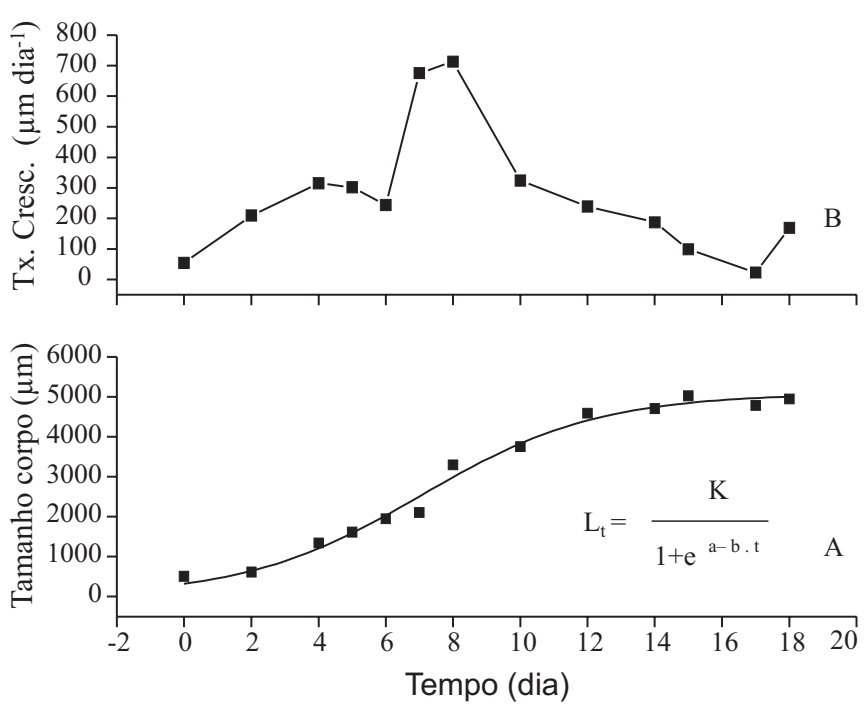

Fig. 4. Goeldichironomus luridus Trivinho-Strixino \& Strixino. A. Curva de crescimento da população nas condições de laboratório (21$\left.26^{\circ} \mathrm{C}\right)$; B. Taxa de crescimento diário das larvas $\left(\mathrm{n}_{2}-\mathrm{n}_{1} / \mathrm{t}_{2}-\mathrm{t}_{1}\right)$. Lt (comprimento médio no instante $\mathrm{t}$ ); $\mathrm{K}$ (tamanho máximo alcançado pelas larvas); t (tempo em dias); a e b são constantes.
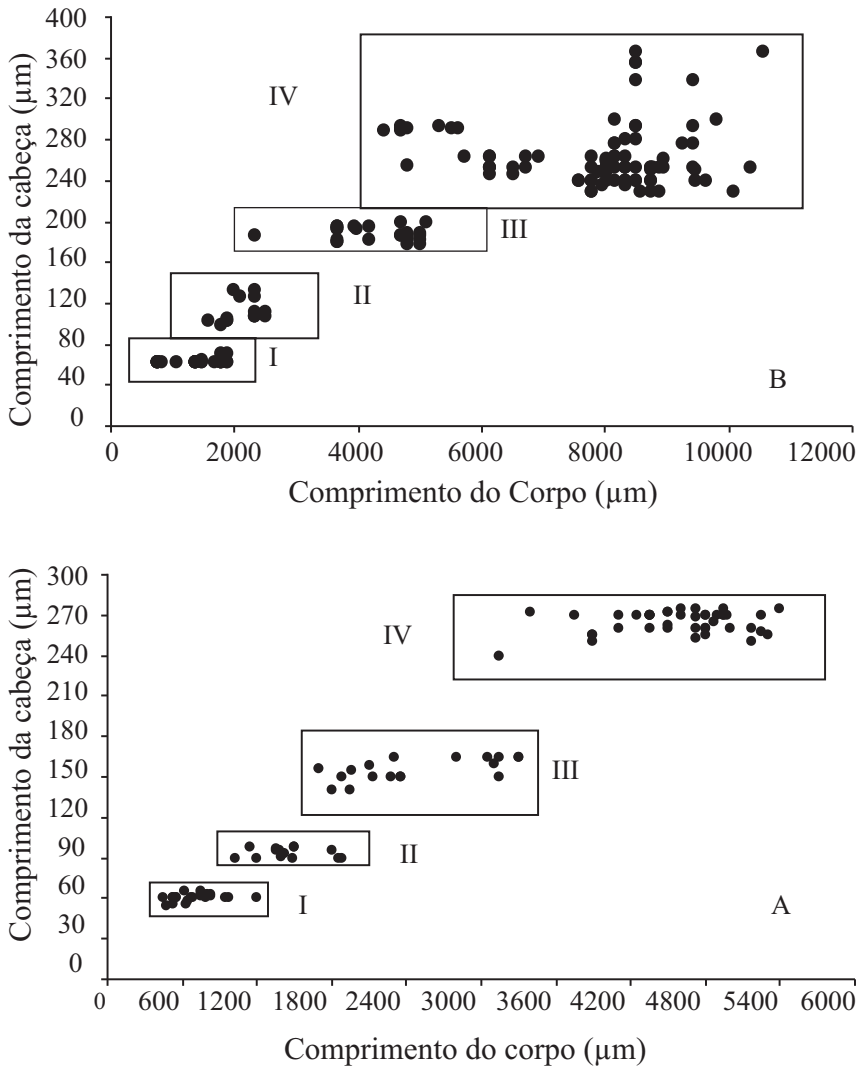

Fig. 5. Relação entre o comprimento ventral da cápsula cefálica $(\mu \mathrm{m}) \mathrm{e}$ comprimento do corpo $(\mu \mathrm{m})$ nos 4 estádios larvais. A: Goeldichironomus luridus Trivinho-Strixino \& Strixino; B: Goeldichironomus maculatus Strixino \& Strixino.

estádios: I estádio (3 dias), o II estádio (2 dias), o III (3 dias) e o IV estádio (10 dias) (Fig. 5A e Tab. IA). A aplicação da regra de Dyar evidenciou uma razão de crescimento constante para os estádios larvais com $\mathrm{r}=1,62 \mu \mathrm{m}$.

Estudos sobre o ciclo de vida de Chironomidae na Região Neotropical são escassos (Strixino \& Strixino 1982). No entanto, esses trabalhos podem fornecer elementos importantes para o

Tabela I. Comprimento mínimo e máximo do corpo e comprimento mínimo e máximo ventral da cápsula cefálica (c.c.) das larvas de Goeldichironomus luridus Trivinho-Strixino \& Strixino (A) e Goeldichironomus maculatus Strixino \& Strixino (B), e duração média dos 4 estádios larvais.

\begin{tabular}{lcccc}
\hline Estádio & $\begin{array}{c}\text { Compr. do Corpo } \\
\text { (MICRA) }\end{array}$ & $\begin{array}{c}\text { Compr. Ventral } \\
\text { da c.c. (MICRA) }\end{array}$ & $\begin{array}{c}\text { Duração Média } \\
\text { (dias) }\end{array}$ & \\
\hline I & $900-1688$ & $60-64$ & 6 & \\
II & $1750-5980$ & $76-142$ & 3 & B \\
III & $2490-6330$ & $180-201$ & 5 & \\
IV & $4410-9459$ & $225-300$ & 14 & \\
\hline
\end{tabular}

\begin{tabular}{|c|c|c|c|}
\hline Estádio & $\begin{array}{l}\text { Compr. do Corpo } \\
\text { (MICRA) }\end{array}$ & $\begin{array}{c}\text { Compr. Ventral } \\
\text { da c.c. (MICRA) }\end{array}$ & $\begin{array}{l}\text { Duração Média } \\
\text { (dias) }\end{array}$ \\
\hline I & $510-975$ & $60-63$ & 3 \\
\hline II & $1050-1800$ & $90-98$ & 2 \\
\hline III & $2100-3450$ & $150-165$ & 3 \\
\hline IV & $3750-5080$ & $255-270$ & 10 \\
\hline
\end{tabular}


conhecimento sobre a bionomia das espécies dessa família e seus aspectos populacionais. Podem ainda esclarecer modificações temporais do ambiente gerar resultados importantes para estudos sobre toxicidade utilizando larvas como indicadoras.

O curto período incubação observado para essas duas espécies de Goeldichironomus (48 horas, entre $21^{\circ}-26^{\circ} \mathrm{C}$ ), aliado à facilidade na obtenção das massas ovígeras no campo e fácil criação em laboratório, com rápido crescimento larval e baixa mortalidade, são interessantes para estudos futuros enfocando efeitos de toxicidade. Como apontado por Nolte (1993), algumas desovas de Chironomidae são de difícil visualização, pequenas e algumas vezes são encontradas apenas em substratos submersos no ambiente aquático. Da mesma forma, algumas espécies apresentam exigências fisiológicas bem definidas e de difícil repetição em condições de laboratório como, a presença de troncos como substrato, altos teores de oxigênio dissolvido, circulação da água constante e exigências alimentares pouco conhecidas o que as tornam menos práticas para estudos dessa natureza.

Agradecimentos. Agradecemos ao Prof. Irineu Bianchini pelo auxílio no programa estatístico e Vanessa Colombo pelas sugestões. A CAPES pelo apoio financeiro.

\section{REFERÊNCIAS}

Fittkau, E. J. 1965. Revision der von E. Goeldi aus dem Amazonasgebiet beschriebenen Chironomiden (Diptera). Chironomidenstudien X. Beitr. Neotropical Fauna 4: 209-226.

Ford, J. B. 1959. A study of larval growth, the number of instars and sexual defferentiation in the Chironomidae (Diptera). Proceedings Real Entomology Society London (A) 31: 151-160.

Krüger, F. 1973. Zur Mathematik dês tierischen Wachstums. II. Vergleich einiger Wachstumsfunktionen. - Helgoländer Wiss. Meeresunters. 25: 509-550.

Nolte, U. 1993. Egg masses of Chironomidae (Diptera). A review including new observations and a preliminary key. Entomologica scandinavica Supplement $\mathrm{n}^{\circ}, 43.75 \mathrm{p}$.

Sander, K. 2000. Chironomidae embryology in the $19^{\text {th }}$ century: Insights and errors of its pioneers, especially August Weismann (1834-1914), and some $2^{\text {th }}$ century sequels. Late $20^{\text {th }}$ century research on Chironomidae: an Anthalogy from the $13^{\text {th }}$ International Symposium on Chironomidae, pp. 1-16.

Strixino, S. T. 1973. A largura da cabeça na determinação das fases larvais de Chironomidae na Represa do Lobo. Dissertação de Mestrado. Universidade de São Paulo (USP).

Strixino, S. \& G. Strixino. 1982. Ciclo de vida de Chironomus sancticaroli Strixino \& Strixino (Diptera, Chironomidae). Revista Brasileira de Entomologia. 26: 183-189.

Strixino, S. \& G. Strixino. 1991. Nova espécie de Goeldichironomus Fittkau (Diptera, Chironomidae) do Brasil. Revista Brasileira de Entomologia. 35: 593-602.

Trivinho-Strixino, S. \& G. Strixino. 1998. Goeldichironomus neopictus, a new species from the Southeast of Brazil: description and bionomic information (Insecta, Diptera, Chironomidae). Spixiana 21: $271-278$

Trivinho-Strixino, S. \& G. Strixino. 2005. Two new species of Goeldichironomus Fittkau from southeast Brazil (Diptera, Chironomidae). Revista Brasileira de Entomologia. 49: 441-445. 